tion through it. This was treated by partial gastrectomy and a short time later retrograde intussusception recurred. In this case the efferent loop again was the intussuscepting one while the afferent loop and the stomach were distended.

At this point it should be stressed that retrograde intussusception of the efferent loop is a rare condition, and any explanation of its inechanism must take cognizance of this fact. Retrograde peristalsis is much less common than iso-peristalsis in the efferent loop. The presence of even transient intussusception at the site of retrograde peristalsis is probably not common and the vast majority of these are spontaneously reduced. This would account for the rarity of the condition. If the absence of sphinicter at the gastro-enterostomy were an important factor one would expect this condition to be much more common than it actually is.

\section{Summary}

I. Retrograde intussusception of the efferent loop of a gastro-enterostomy is a well-recognized, although infrequent condition. No previous attempt has been made to explain its mechanism.

2. Another case has been reported-this time of a thrice-occurring retrograde intussusception which was successfully treated surgically.
3. A theory to explain the mechanism of retro-气 grade intussusception through a gastro-enterostomy stoma is put forward.

4. Some rare and interesting cases of retrograde. intussusception without gastro-enterostomy are reviewed.

\section{BIBLIOGRAPHY}

ALVAREZ, W. C. (1933), Proc. Staff Meeting, Mayo Clin., 10, 103त 'Introduction to Gastro-Enterology,' (1948), Heineman.

BROGLIO, R. (1946) 'Invagination of Duodenum into th Stomach,' Gio Sc. Med. N.8.1; Auguist, ro-I I.

BEST \& TAYLOR, 'Physiological Basis of Medical Practice,' ch $\vec{\nabla}$ 43-44.

CANNON, W. B. (1898), Movements of Stomach studied by $\overrightarrow{4}$ Rontgen Rays,' Amer. Physiol., 1, 359; ' Emptying of Humarw Stomach ' (1904), 10, xix.

GROPER (1946), Ann. Surg., September, 112, 344.

FITZWILLIAMS, D. C. (1908), Lancet, i, 628, 707.

HENDERSON, V. E. (1928), 'Smooth Muscle Experiment Amer. F. Physiol., 86, 82.

IBOS and LEGRAND DESMOS (1929), Bull. \& Mem. Soc. Nat de Chir., 55, 1277 .

KLEINE, L. (1925), Arch. Surg., 12, 571.

LEWIS E. E (1936) 'Retrograde Colo-Colic Invagination Brit. $\mathscr{f}$. Surg., 23, 683.

MCNAMARA (1944), 'Retrograde Jejuno-Gastric Intussusception through a Sub-total Gastrectomy Stoma,' Ann. Surgery August, 120, 207. THOMAS, CRIDER and MOGAN (1934), Amer. F. Physiol. $\vec{D}$
ro8, 683.

THOREK, P. (1947), F.A.M.A., Jänuary 4, 133, $21-23$.

WU, H. K., and CHANG, K. H. (1944), 'Acute RetrogradeIntussusception of Jejunum through a Gastro-Enterostomxo Stoma in the Adult,' Chinese med. $F$., Chengtu Edition, Octooger 63A, 26-29.

\title{
RECURRENT RETROGRADE GASTRO-JEJUNAL INTUSSUSCEPTION
}

\author{
By W. K. Dougläs, F.R.C.S. \\ Senior Surgical Registrar, Manchester Royal Infirmary
}

Since igiz; when the first case of retrograde gastro-jejuinal intussúsception was published (Steber, 19r7); many cases have been recorded. However; only one other case of recurrent retrograde gastro-jejunal intussusception has been reported (Baumatin; rizi). Therefore the following case is of special interest.

\section{Case Report}

History: A female, aged 47, was admitted to the Manchester Royal Infirmary on April 18, 1952. with symptoms of a duodenal ulcer. Barium meal confirmed the clinical diaghosis. Operation was performed the following day. An ulcer was found in the first part of the duodenum. which was producing some degree of stenosis. A fractional gastric analysis had shown a low acid curve. $A$ 응 posterior gastro-enterostomy was performed.

The pätient made an uninterrupted recovery and was discharged home on May 1, 1952.

On July 8, 1952, the patient was re-admitted with a history that she had been quite well untif the day before, when she was awakened at night with a heavy gnawing pain in the epigastrium The pain was constant and continued to increase ${ }^{\omega}$ in severity. Vomiting commenced soon after tho onset of pain. Vomiting relieved the pain and was profuse and blackish-brown in appearance. It wase effortless and smelt foul. She had nothing to eat all day and had no bowel action.

On admission there was some degree of abdominal distension with guarding and tender $\frac{?}{\square}$ 
ness over the epigastrium. No mass was palpable. The tongue was dry and furred. A provisional diagnosis lay between, exacerbation of the duodenal ulcer or upper small bowel obstruction.

The patient was kept under observation for 48 hours. A Ryle's tube was passed and an intravenous saline drip set up.

By July 10, 1952, as the patient's condition had not settled, a laparotomy was carried out. The abdomen was opened through a left paramedian incision. The duodenal ulcer showed signs of healing. Intussusception of the efferent loop through the gastro-enterostomy stoma was found. Manual reduction was effected quite easily. Following this it was found that about $3 \mathrm{ft}$. of the efferent loop had been involved. The site of the commencement of the intussusception was difficult to determine, but it was thought that it had begun about 2 in. from the stoma. The affected loop was found to be bruised, but otherwise quite healthy. The afferent loop was distended. In an attempt to prevent recurrence, the efferent loop of jejunum was sutured to the transverse colon for about 3 in.

The patient made an uninterrupted recovery and was discharged on July 22, $195^{2}$.

On October 25, 1952, the patient was seen in the outpatient department and found to be perfectly well.

On December 10, 1952, she was re-admitted to hospital with a history of sudden onset of epigastric pain at 2 a.m. that morning. She vomited at the onset and every ro minutes afterwards. Pain was relieved by vomiting to some extent. The vomit was bile-stained and contained no blood. The physical signs were very similar to those elicited on her previous examination, and in view of the similarity of the clinical picture an immediate laparotomy was carried out.

The abdomen was opened through a right upper paramedian incision. A number of adhesions had to be divided before the stomach could be exposed. The lesser sac was opened through the great omentum. It was found that the efferent loop had intussuscepted through the stoma for about $3 \mathrm{ft}$. No trace was left where, at the previous operation, the transverse colon had been hitched to the efferent loop. The intussusception was easily reduced and again the transverse mesocolon was sutured to the efferent loop for about 4 in. This manoeuvre was carried out as, at the time of operation, I was unaware that this had previously been tried.

The patient made an uninterrupted recovery and was discharged on December 24, $195^{2}$.

\section{Discussion}

The aetiology of this condition has always been a puzzle. Drummond expressed the view that a wide stoma may cause rapid emptying which may result in irritation of the jejunal mucous membrane as a result of its contact with food, and so a jejunitis results. Radiological examination demonstrated the irritability of the jejunal wall, and it was suggested that there, areas of jejunitis may set up waves of antiperistalsis resulting in the formation of an intussusception (Drummond, r923).

Since 1917 when the first case was published (Steber, I917) many theories have been put forward but none have received substantial recognition.

Shackman has given a useful classification of the types of intussusception met with. He divides them into three types (Shackman, I940).

Type I. Intussusception of the afferent loop.

Type 2. Intussusception of the efferent loop.

Type 3. Intussusception of both loops.

This case comes under the second and most commonly met with type.

The onset of the condition was classically sudden, and the early appearance of altered blood in the vomit was equally characteristic, though there was no evidence of frank blood in the vomit, a feature seen in many other cases published.

James Moroney (1948) records a case or a man of 50 who had a severe haematemesis in association with the intussusception of an efferent loop through a gastro-enterostomy stoma. The intussuscepted loop had undergone gangrene and had to be resected. The diagnosis was made late because the haematemesis was such a predominant feature that it had masked the underlying cause.

It is said that in $5^{\circ}$ per cent. of the cases a swelling is palpable in the epigastrium. In spite of the absence of marked rigidity or distension in this case, no swelling could be felt.

Baumann (1921) in his recorded case, notes that there was an interval of only eight weeks between the first and second operation; as distinct from the average time of six years between the performance of a gastro-enterostomy and the occurrence of the intussusception. In this case there was a gap of only three months between all three operations.

An interesting possibility has been noted by Sibley (1934). He states that there are two main types of intussusception. Acute and chronic. The acute form is the type generally met with and may occur any time from five weeks to 14 years after the original operation. The chronic form, however, is intermittent in nature and in a number of cases may pass unnoticed. He described a typical case. A patient attended complaining of a feeling of constriction in the mid-epigastrium and an inability to bear anything tight round his waist. These symptoms occurred immediately after a meal and lasted from one to three hours. During the screening of the stomach in one of these 
attacks the efferent loop of jejunum was seen to enter the stomach. The patient underwent an immediate operation but no evidence of an intussusception was found. It had evidently undergone spontaneous reduction.

Cases have been reported following partial gastrectomy as well as following gastro-enterostomy, Allen E. Grimes (1949) and W. L. McNamara (1944). They made the observation that there seemed to be no correlation between the incidence of intussusception and the size of the stoma. That would appear to be a widely held opinion.

Shackman has made a plea for early diagnosis. He states that of those cases not operated on within the first 24 hours 50 per cent. die. As has previously been noted, early diagnosis can be extremely difficult. When a patient develops recurrent attacks of colicky epigastric pain with repeated blood-stained vomit, combined with upper abdominal distension and absence of rigidity, the diagnosis is relatively easy, but may have been made too late. The condition should be considered in any patient who complains of abdominal pain and vomiting following a gastroenterostomy.

\section{Treatment}

It is now generally realized that provided the condition is recognized early enough, manual reduction of the intussusception can be carried out at operation without any difficulty, and the results will be satisfactory in all cases.

Before the first operation in this case there was a delay of 36 hours. However, the patient did not develop the severity of vomiting which develops in cases where operation is delayed, mainly due to the fact that she had a Ryle's tube down, which was aspirated hourly, and her fluid intake was maintained by intravenous infusions of isotonie saline and 5 per cent. dextrose. On both occasion there was no difficulty in manually reducing the intussusception, but in both cases an attempt was: made to prevent recurrence. Because of lack of previous knowledge it turned out that I had pere formed the same manoeuvre which had bee carried out at the first operation, namely the hitch ing up of 4 in. of the efferent loop of the jejunum to the transverse colon. Therefore there seems to bof no reason why she should not develop an intussusception for the third time.

Various measures have been suggested for pre $\overrightarrow{\vec{\sigma}}$ venting recurrence, namely:

I. Undoing the gastro-enterostomy and reforne ing it.

2. Entero-enterostomy between the afferent ang efferent loops.

3. Partial gastrectomy.

Naturally, as this is only the second case of res current gastro-jejunal retrograde intussusception to be reported, it is very difficult to judge the efficacy of these methods.

Shackman reported the occurrence of a cas even though entero-enterostomy had been carrief out at the time of the gastro- enterostomy.

My thanks are due to Mr. D. McK. Suthe land, under those care the case was admitted, 竞芭 permission to publish this article.

\section{BIBLIOGRAPHY}

BAUMANN, Arch. Klin. Chir., II1, 504.

DEBENHAM, R. K. (1935), B.M.f., 1, 250.

GRIMES, ALLEN E. (1949), Ibid., 129, 404.

HAMILTON DRUMMOND (1928), B.F.S., 11,79 .

MCNAMARA, W. L. (1944), Ann. of Surg., 120, 207.

MORONEY, J. (1948), Ibid., 35, 374 .

SHACKMAN, R. (1940), B.f.S., 27, 475.

SIBLEY (1934), Proc. Staff Mavo Clinic, 9, 25.

STEBER (1917), Muench. Med. Wchschr., 64, 648.

\section{NON-OSTEOGENIC FIBROMA OF BONE \\ By RAYMOND FARRow, F.R.C.S. \\ Surgical Registrar, Westminster Children's Hospital, London}

The term non-osteogenic fibroma of bone was first used by Jaffe and Lichtenstein in 1942 in order to describe a benign neoplasm of bone which, in their opinion exhibited characteristic clinical, radiological and pathological features. They defined the lesion as a benign tumour derived from mature marrow connective tissue which showed no evidence of ossification. In the latter respect it becomes distinguishable from other fibromas ot bone in which evidence of osseous metaplasia is always present and it is for this reason that the prefix ' non-osteogenic' has been employed.

\section{Clinical Appearances}

All cases hitherto recorded have occurred be윰 tween the ages of 6 and 21 years and with the 